\title{
Thermochemical emission and transformation of chlorinated paraffins in inert and oxidizing atmospheres
}

\author{
Shanzhi Xin ${ }^{\text {a, b }}$, Wei Gao ${ }^{\text {a, d }}$, Yawei Wang a, c, d, * , Guibin Jiang ${ }^{\text {a }}$ \\ a State Key Laboratory of Environmental Chemistry and Ecotoxicology, Research Center for Eco-Environmental Sciences, Chinese Academy of Sciences, \\ Beijing 100085, China \\ ${ }^{\mathrm{b}}$ Hubei Key Laboratory of Industrial Fume and Dust Pollution Control, Jianghan University, Wuhan 430056, China \\ ${ }^{c}$ Institute of Environment and Health, Jianghan University, Wuhan 430056, China \\ ${ }^{\mathrm{d}}$ University of Chinese Academy of Sciences, Beijing 100049, China
}

\section{H I G H L I G H T S}

- CP52 was decomposed by a one-step process at $210-330{ }^{\circ} \mathrm{C}$ with $\sim 92 \mathrm{wt} \%$ weight loss.

- SCCPs gradually released into the gas phase and decomposed completely at temperatures $>400{ }^{\circ} \mathrm{C}$.

- Thermal decomposition of CP52 generated high concentrations of PAHs and Cl-PAHs.

- PAHs and $\mathrm{Cl}-\mathrm{PAHs}$ were dominated by 2- to 5-ring and mono-CBz, PCBs, and PCNs, respectively.

\section{A R T I C L E I N F O}

Article history:

Received 3 June 2017

Received in revised form

4 July 2017

Accepted 4 July 2017

Available online 5 July 2017

Handling Editor: J. de Boer

Keywords:

Thermochemical

MCCPs

SCCPs

Chlorinated aromatic hydrocarbons
G R A P H I C A L A B S T R A C T

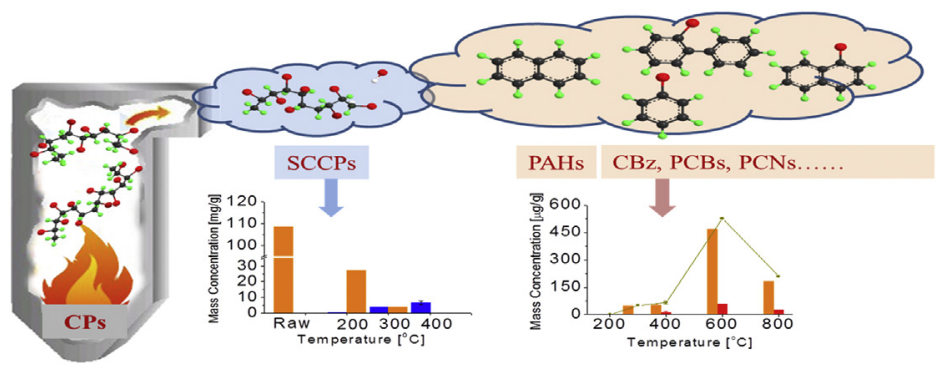

\begin{abstract}
A B S T R A C T
Chlorinated paraffins (CPs) generally function as flame retardants and plasticizers in various materials. They are most likely to be processed by thermal processes during the entire life cycle. However, data on the formation and emission of CPs during thermal processes are still not fully understood. In this study, we simulated industrial thermal processes to investigate the emission of medium-chain chlorinated paraffins (MCCPs) and short-chain chlorinated paraffins (SCCPs) using commercial CP52 as the feedstock. We found that CP52 decomposed very easily at $210-320^{\circ} \mathrm{C}$. The decomposition of CPs generated large quantities of MCCPs and SCCPs. These remained in the residue at low temperature $\left(200^{\circ} \mathrm{C}\right)$ and were gradually released into the gas phase at higher temperatures. MCCPs and SCCPs were not detected in either the residue or the gas phase when the temperature exceeded $400{ }^{\circ} \mathrm{C}$. However, considerable concentrations of aromatic and chlorinated aromatic hydrocarbons (Cl-PAHs) were identified in the gas phase, and they were formed as the amount of SCCPs and MCCPs decreased. Cl-PAHs were dominated by low-chlorinated chlorobenzenes, polychlorinated biphenyls, and polychlorinated naphthalenes. Oxygen promoted the release and decomposition of SCCPs in the gas phase. The results of the present study revealed the release of MCCPs and SCCPs and their synergistic emission with $\mathrm{Cl}$-PAHs when CPs were subjected to heat. This work may also provide data for developing multiple techniques to control the emission of CPs and Cl-PAHs.
\end{abstract}

๑) 2017 Elsevier Ltd. All rights reserved.

\footnotetext{
* Corresponding author. State Key Laboratory of Environmental Chemistry and Ecotoxicology, Research Center for Eco-Environmental Sciences, Chinese Academy of Sciences, P.O. Box 2871, Beijing 100085, China.

E-mail address: ywwang@rcees.ac.cn (Y. Wang).
} 


\section{Introduction}

Chlorinated paraffins (CPs), also called polychlorinated n-alkanes, are a group of chlorinated organic mixtures with a chlorination degree of 30-70\% and carbon atom number ranging from 10 to 30 (Bayen et al., 2006). CPs can be subdivided into short- (SCCPs, $\mathrm{C}_{10-13}$ ), medium- (MCCPs, $\mathrm{C}_{14-17}$ ), and long-chain CPs (LCCPs, $\mathrm{C}_{18-30}$ ) (Thompson and Vaughan, 2014). Among the CP groups, SCCPs have attracted extensive attention because of their properties similar to those of persistent organic pollutants (POPs) (Houde et al., 2008; Zeng et al., 2011; Ma et al., 2014b). SCCPs have been detected in various environmental matrices, biota, and even in the Arctic regions (Zeng et al., 2012; Hilger et al., 2013; Ma et al., 2014a; Diefenbacher et al., 2015; Zeng et al., 2015; Gao et al., 2016a; van Mourik et al., 2016; Wang et al., 2017), and humans have a high risk when exposed to SCCPs (Iino et al., 2005; Ma et al., 2014b; Xia et al., 2016; Cao et al., 2017). Recently, the Persistent Organic Pollutants Review Committee (POPRC) of the Stockholm Convention has recommended the listing of SCCPs in Annex A to the Convention (POPRC, 2017).

In China, commercial CPs labeled CP52 contributed to over $80 \%$ of CP consumption annually. The chlorination degree of CP52 was $\sim 52 \%$, and the carbon chain length ranged from $C_{12}$ to $C_{18}$ (Zhang et al., 2013). They were commonly used as flame retardants and plasticizers in polymers such as plastics, textiles, and rubber (van der Gon et al., 2007; Feo et al., 2009). The CP-containing polymers were widely used in decorations, packing, and functional materials in the household, marketplace, and industrial facilities (Yu et al., 2016). As SCCPs do not have natural sources so far, they can release into the environment during the production, storage, use, and disposal of SCCPs and SCCP-containing products. Studies have found that during $\mathrm{CP}$ production, plasticizer- or flame-retardant synthesis, and the usage of $\mathrm{CP}$-containing materials, the emission of SCCPs was 1440-1788 tons in 2011, contributing approximately $2.3 \%$ of the total SCCPs produced (Xu et al., 2014; Jiang et al., 2017). Therefore, most of the SCCPs remained in the polymers that contain CPs. In other words, the transformation and emission of SCCPs was determined by the usage or disposal of these polymers.

During the life cycle of these materials that contain CPs, they are most likely to experience thermal processes, such as incineration, opening burning and accidental compartment fire. It might release SCCPs into the environment unintentionally during these processes. It is estimated that the production of plastic waste exceeded 280 million tons in 2011 and is increasing exponentially (Kaimal and Vijayabalan, 2016). It can be inferred that large quantities of SCCPs might be released unintentionally into the environment during thermal processes.

Thus far, few studies have investigated the thermal degradation of CPs with different chlorine content (Sosa, 1975; Camino and Costa, 1980, 1981; Bergman et al., 1984). Sosa synthesized four chloroparaffins with $18-38 \%$ chlorine and found that they were completely volatilized at around $330^{\circ} \mathrm{C}$ (Sosa, 1975). CPs with $70 \mathrm{wt}$ $\%$ chlorine underwent dehydrochlorination at $250{ }^{\circ} \mathrm{C}$, and $60-70 \%$ chlorine was released into the environment. The remaining chlorine in residue was completely dehydrochlorinated at $300-600{ }^{\circ} \mathrm{C}$ (Camino and Costa, 1980). Bergman et al. analyzed the degradation products of polychlorinated alkanes with $59 \%$ and $70 \%$ chlorine by GC-MS. They found that aliphatic, aromatic, and polychlorinated aromatic hydrocarbons were the major products (Bergman et al., 1984). However, as compared to these conventional compounds, rare attention has been paid on the formation and emission of SCCPs and MCCPs during the thermal decomposition of CPs thus far. The lack of such data limits the establishment of emission inventories and the risk assessment of CPs. However, it should be realized that as a source of carbon, hydrogen, and chlorine, CPs might also generate aromatic and chlorinated aromatic hydrocarbons (Cl-PAHs) during thermochemical processes (Oh et al., 2007; Poddar et al., 2013). A previous study indicated that the decomposition of CPs could generate polychlorinated benzenes, biphenyls, and naphthalenes (Bergman et al., 1984). Thus, the synergistic effects between SCCPs and Cl-PAHs are also critical to evaluate the environmental risk of CPs and develop multiple emission control techniques.

Therefore, in this study, we simulated industrial thermal processes in a laboratory-scale furnace. The objective of this study is to investigate the formation and emission characteristics of CPs and the composition and congeners of the resultant products. The results of this work are helpful to obtain a brief understanding of the synergistic effects between the emission of SCCPs, MCCPs, and $\mathrm{Cl}$ PAHs during thermal processes. The ultimate goal is to develop multiple technologies to control their formation.

\section{Experimental section}

\subsection{Feedstock and reagents}

Commercial CP52 was selected as the feedstock in this study, and it was in the form of transparent viscous oil. Prior to the experiment, the characteristics of CP52 were analyzed according to our previous method by quadrupole time-of-flight high-resolution mass spectrometry (GC-QTOF-HRMS) with a chemical ionization source working in the negative ion mode (Gao et al., 2016b). The proportion of SCCPs and MCCPs in the commercial CP52 was $10.9\left(108.5 \mathrm{mg} \mathrm{g}^{-1}\right)$ and $85.5 \mathrm{wt} \%$ ( $855.4 \mathrm{mg} \mathrm{g}^{-1}$ ), respectively. The dominant carbon and chlorine atom homologs of SCCPs and MCCPs were $\mathrm{C}_{13}, \mathrm{C}_{14}$ and $\mathrm{Cl}_{7-8}$, $\mathrm{Cl}_{8}$, respectively (Fig. S1). Finally, the ash content in CP52 was determined to be approximately zero, according to ASTM standards (D1102-84 (2007)). The reagents dichloromethane and hexane were of pesticide analytical grade and supplied by J.T. Baker (Philipsburg, USA). A solution of the SCCP mixture (100 ng L ${ }^{-1}, \mathrm{C}_{10}-\mathrm{C}_{13}$ with $51 \%$, $55.5 \%$, and $63 \%$ chlorination, $100 \%$ purity) in cyclohexane and $\varepsilon$-hexachlorocyclohexane $\left(\varepsilon-\mathrm{HCH}\right.$, solution in cyclohexane, $10 \mathrm{ng} \mathrm{L}^{-1}, 99.9 \%$ purity) were purchased from Dr. Ehrenstorfer GmbH (Augsburg, Germany) (Gao et al., 2016b).

\subsection{Thermal kinetics of CP52}

The decomposition characteristic of CP52 was investigated using a thermogravimetric analyzer (TGA 4000, Perkin Elmer) under nitrogen and air. For each experiment, approximately $20 \mathrm{mg}$ of CP52 was evenly spread in a crucible and heated from room temperature to $450{ }^{\circ} \mathrm{C}$ at six different heating rates $\left(2,5,10,20,50\right.$, and $100{ }^{\circ} \mathrm{C}$ $\mathrm{min}^{-1}$ ) with a gas flow rate of $80 \mathrm{~mL} \mathrm{~min}^{-1}$ and then held for $3 \mathrm{~min}$ at the final temperature. Each experimental result was tested at least twice to ensure repeatability. The activation energy of CP52 thermal decomposition was calculated from the TGA data (Supplementary Materials, Part S1) by the Flynn-Wall-Ozawa method.

\subsection{Combustion setup and procedures}

The combustion experiments were performed on a bench-scale furnace, and the details are given in Fig. S2. In this study, the reaction temperature was set at $200,300,400,600$, and $800{ }^{\circ} \mathrm{C}$ to investigate the product composition at different temperatures. The carrier gas was a high-purity dry air and nitrogen (99.999\%) with a flow rate of $150 \mathrm{~mL} \mathrm{~min}{ }^{-1}$ to simulate combustion (oxidizing atmosphere) and pyrolysis (inert atmosphere). Prior to each trial, the furnace was heated to the preset temperature with nitrogen or air and stabilized for $10 \mathrm{~min}$. After that, $500 \pm 10 \mathrm{mg}$ CP52 was placed in the sample basket with a thin layer and then placed at the center 
of the reactor. The pyrolysis or combustion started as soon as CP52 entered the reactor. The carrier gas flowed through the bottom of the quartz tube and carried the volatiles from the furnace to the collection system. The hot volatile mixture first entered the Ushape precooling glass tube, where the gas mixture was cooled to ambient temperature, and some condensable compounds were collected. The noncondensable volatiles and gaseous products were then absorbed by the absorption bottle with $50 \mathrm{~mL}$ absorption liquid (mixture of dichloromethane and hexane $(1: 1, v / v)$ ). After $30 \mathrm{~min}$, the pyrolysis or combustion experiment was completed. The quartz tube was removed from the furnace and cooled to room temperature with the carrier gas.

\subsection{Product collection, analysis, and quantification}

After each experiment, the system was cooled down to ambient temperature under $\mathrm{N}_{2}$ or air atmosphere. The quartz reactor and glass tube were washed three times with a 50-mL mixture of dichloromethane and hexane. After the absorption liquid was removed, the absorption bottles were also washed three times using the $50-\mathrm{mL}$ mixture of dichloromethane and hexane. The condensable compounds in glass tube, absorption liquid, and the washing solution were mixed together and collectively called the volatile solution (VS). The VS was then concentrated on a rotary evaporator to approximately $2 \mathrm{~mL}$ for analysis. The char or ash residues were collectively denoted as the solid residue (SR). The SR was collected and extracted by a mixture of dichloromethane and hexane under microwave and then filtered. The filtrate was analyzed according to the following procedure.

Prior to the analysis, $10 \mathrm{ng}$ of $1,5,5,6,6,10$-hexachlorodecane $\left({ }^{13} \mathrm{C}_{10}\right)$ in a $100-\mu \mathrm{g} \mathrm{mL} \mathrm{m}^{-1}$ solution in cyclohexane (Andover, USA) was added as the internal standard to quantify the SCCPs. The composition of the SCCP congeners in the VS was analyzed using an Agilent 7200 GC-QTOF mass spectrometer (Agilent Technologies, Santa Clara, USA) operated in the negative chemical ionization mode and controlled by MassHunter Acquisition B.07. The quantification method was detailed in our recent work (Gao et al., 2016b). An electron ionization source was used to identify and quantify the organic compounds in the VS. The mass spectra of the compounds were identified by matching the respective GC chromatograms with the NIST library. For the chlorinated aromatic compounds, chlorobenzenes $(\mathrm{CBz})$, polychlorinated biphenyls (PCBs), and polychlorinated naphthalenes (PCNs) were analyzed qualitatively by the external standard method. Standard solutions of CBz, PCBs, and PCNs were purchased from SUPELCO company (Zhang et al., 2014b), Wellington Laboratories, and Cambridge Isotope Laboratories (Jiang et al., 2015), respectively. The GC oven temperature program is given in the Supplementary Materials (Part S2). The identification and quantification of the compounds were performed by a combination of untargeted (deconvolution of mass spectra chromatograms) and targeted (quantification of identified chemicals). The deconvolution and quantification procedure was similar to that used in a previous study (Zhang et al., 2014a).

\subsection{Quality assurance and quality control}

To avoid possible contaminants, the quartz reactor and sample basket were calcined at $800{ }^{\circ} \mathrm{C}$ in the presence of air for $1 \mathrm{~h}$ before and after the experiment. Three runs were performed under inert and oxidizing atmospheres at $400{ }^{\circ} \mathrm{C}$ to evaluate the reproducibility of the experiment. It was found that the relative standard deviation of SCCP and MCCP concentration was 3.4-16.6\%, while those of $\mathrm{CBz}$, PCB, and PCN concentrations under inert and oxidizing atmospheres were $8-10.7 \%, 4.3-6.8 \%$, and $5.1-9.3 \%$, respectively. This indicates that the experimental and analytical procedure have an acceptable level of reproducibility. Experimental blanks were conducted at the same conditions without CPs in the sample basket, while procedural blanks were performed for each batch of solvents. No CPs and other Cl-PAHs were detected in these blank tests. The limit of detection (LOD) for the SCCPs and MCCPs was defined as the detection of the two most abundant homologs: $\mathrm{C}_{11} \mathrm{H}_{18} \mathrm{Cl}_{6}$ and $\mathrm{C}_{14} \mathrm{H}_{23} \mathrm{Cl}_{7}$. The LOD of the MCCPs was $30 \mathrm{ng} \mathrm{mL}$ (52\% Cl MCCPs), while that of the SCCPs was $25 \mathrm{ng} \mathrm{mL}^{-1}(55.5 \% \mathrm{Cl}$ SCCPs). During the experiments, a blank test was conducted before the experiment and after every three samples.

\section{Results and discussion}

\subsection{Thermal decomposition kinetics of CPS}

The TGA and derivative thermogravimetric (DTG) curves at a heating rate of $10{ }^{\circ} \mathrm{C} \mathrm{min}-1$ and the activation energy of CP52 decomposition are shown in Fig. 1. It is apparent that the thermal decomposition of CP52 was a one-step process at $210-330^{\circ} \mathrm{C}$ with 92\% weight loss (Fig. 1a). The decomposition of polyvinylchloride occurred between $250{ }^{\circ} \mathrm{C}$ and $320^{\circ} \mathrm{C}$, and the weight loss was approximately 65\% (Jordan et al., 2001; Yu et al., 2016). It was clear that although the decomposition temperature of CP52 largely overlaps with that of high-chlorinated CP and PVC, the initial temperature of CP52 was much lower. In other words, the thermal stability of CP52 was very low, and it was susceptible to thermal decomposition.

The TGA and DTG curves obtained under inert and oxidizing atmospheres were similar, suggesting that the reaction atmosphere exerts a minor effect on the decomposition of CP52. Accordingly, the trend in activation energy with respect to conversion is similar regardless of the reaction atmosphere and fluctuates slightly when the conversion ranges from 0.1 to 0.8 (Fig. $1 \mathrm{~b}$ ). The average activation energy of CP52 was $81.5 \pm 7.8 \mathrm{~kJ} \mathrm{~mol}^{-1}$ in inert atmosphere and $81.7 \pm 7.2 \mathrm{~kJ} \mathrm{~mol}^{-1}$ in oxidizing atmosphere, which is far lower than that of widely used plastic constituents such as PVC, PP, and PE (Castro et al., 2012; Tang et al., 2015; Diaz Silvarrey and Phan, 2016). This finding is reasonable because CP52 has been widely used as a flame retardant, and it decomposes first to block the flame from the surface of polymers. The activation energy of CP52 in the present study was also much lower than the decomposition of $70 \mathrm{wt} \% \mathrm{CP}$ $\left(\sim 167 \mathrm{~kJ} \mathrm{~mol}^{-1}\right)$, as reported by Camino and Costa (1980). This suggests that chlorine content might have affected the decomposition characteristic of different CPs.

\subsection{Formation characteristics of SCCPs and MCCPS}

The total concentration of SCCPs and MCCPs determined in the VS and SR at different temperatures is shown in Fig. 2a-d. With the increase in temperature, the color of raw CP52 turned from transparent to brown at $200{ }^{\circ} \mathrm{C}$, to black at $300{ }^{\circ} \mathrm{C}$, and finally leaving a black char residue at $400{ }^{\circ} \mathrm{C}$. However, there was no residue after the combustion of $\mathrm{CP} 52$ at $600{ }^{\circ} \mathrm{C}$ and above.

At $200{ }^{\circ} \mathrm{C}$, small amounts of SCCPs and MCCPs were detected in VS, but they almost remained in SR. The heating of CP52 resulted in the formation of $2.7-4.2 \mathrm{wt} \%\left(27-42 \mathrm{mg} \mathrm{g}^{-1}\right)$ SCCPs and 76.4-79.2 wt\% (764-79.2 $\mathrm{mg} \mathrm{g}^{-1}$ ) MCCPs in SR under inert and oxidizing atmospheres, respectively. This indicates that the amount of thermally induced emission of SCCPs and MCCPs was very low. However, it should be realized that the amount of SCCPs and MCCPs in the residue was still much lower than that in the raw CP52 sample. The mass change in CP52 was approximately 6-11 wt\%, which demonstrates that at $200{ }^{\circ} \mathrm{C}$, SCCPs and MCCPs decomposed notably rather than volatilizing. This further proves the previous result that CPs are susceptible to temperature (Fig. 1a). 

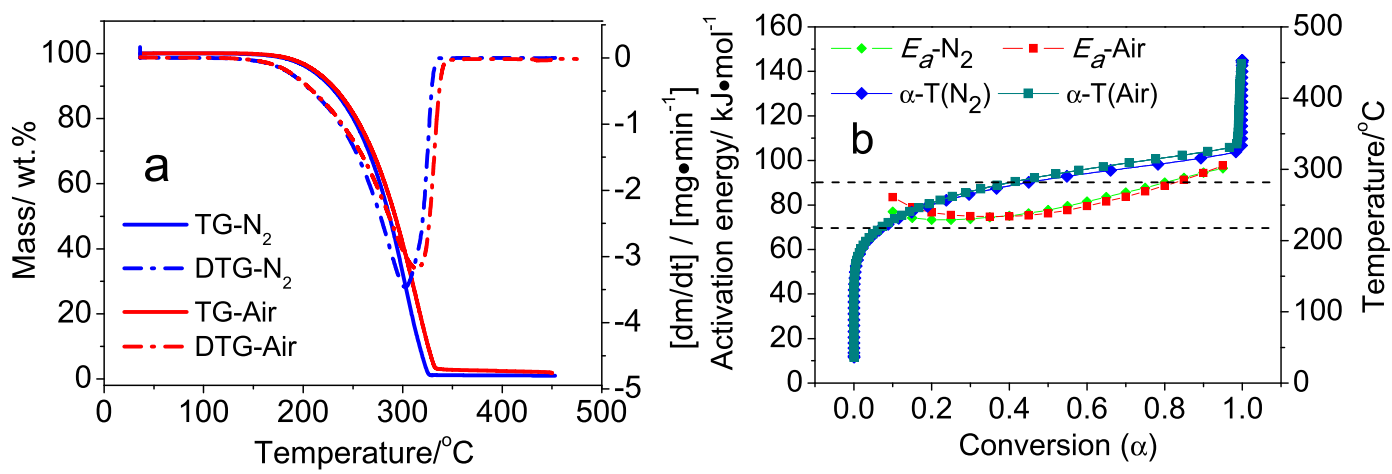

Fig. 1. TGA and DTG curves (a) and the activation energy versus conversion (b) of the thermal decomposition of CP52 under inert and oxidation atmospheres.
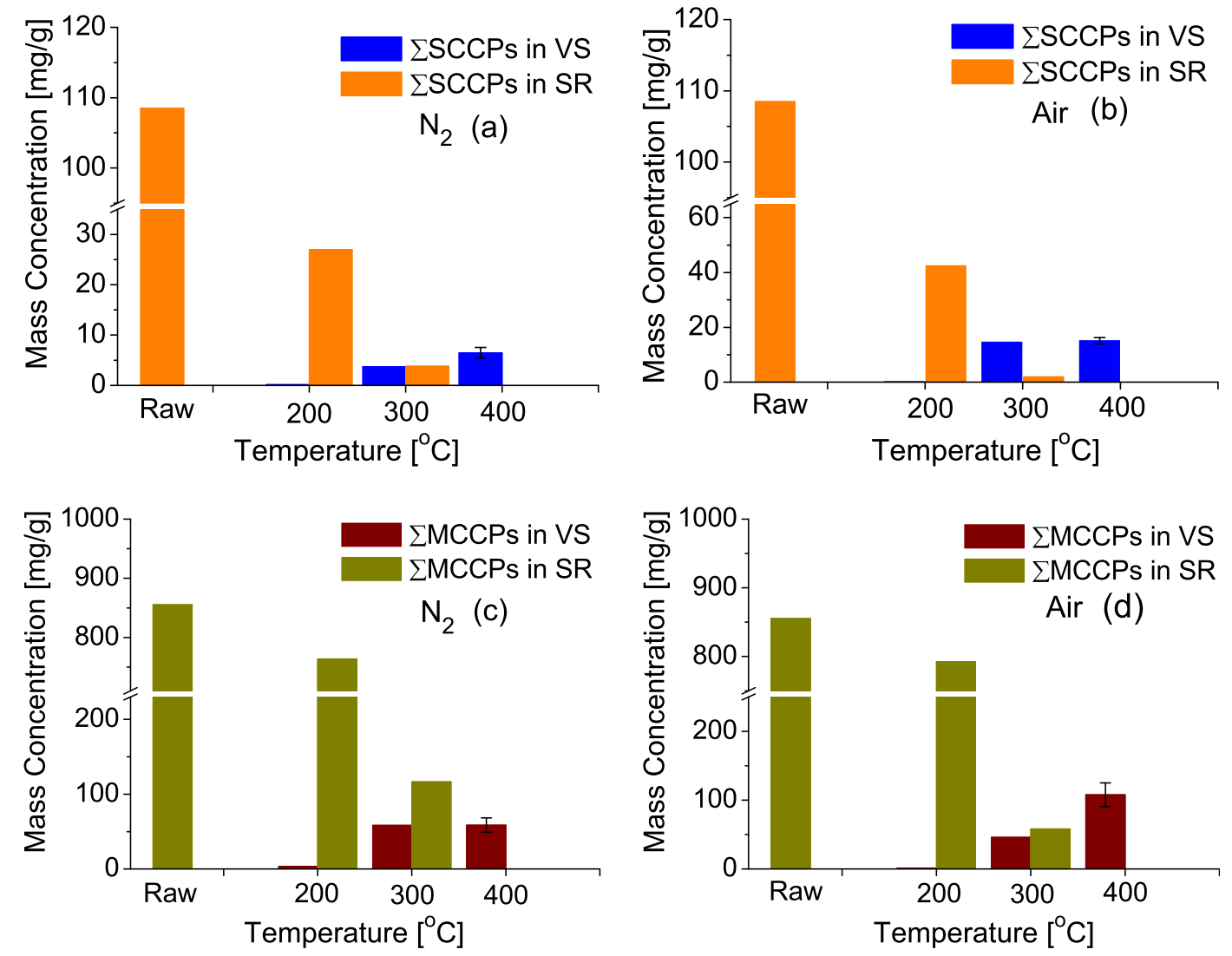

Fig. 2. Comparison of SCCP and MCCP concentrations in VS and SR under (a, c) inert and (b, d) oxidative atmospheres at different temperatures.

As the temperature was increased from $200{ }^{\circ} \mathrm{C}$ to $300{ }^{\circ} \mathrm{C}$, SCCP and MCCP concentrations in the residue decreased rapidly. In contrast, the concentration in the gas phase increased remarkably. This implies that the release of SCCPs and MCCPs was accelerated with the increase in temperature. SCCPs and MCCPs can be further converted to other products by thermal decomposition in the gas phase, such as $<\mathrm{C}_{10}$ hydrocarbons or aromatic compounds. At $400{ }^{\circ} \mathrm{C}$, the SCCPs in the gas phase under inert atmosphere and MCCPs under oxidizing atmosphere increased slightly. Moreover, SCCPs and MCCPs were both undetectable in the residue. As the temperature increased further to $600{ }^{\circ} \mathrm{C}$, SCCPs were not detected in either the residue or the gas phase. Fig. 2a and b shows that the amount of SCCPs under oxidizing atmosphere was approximately two times higher than that under inert atmosphere in SR at $200{ }^{\circ} \mathrm{C}$ and in VS at $300-400^{\circ} \mathrm{C}$. In contrast, from Fig. $2 \mathrm{c}$ and d, the amount of MCCPs under oxidizing atmosphere was lower than that under inert atmosphere both in VS and SR at $200-300{ }^{\circ} \mathrm{C}$. The above results suggest that oxygen facilitates the decomposition of MCCPs.

The carbon and chlorine atom congener distribution patterns of SCCPs and MCCPs in VS under inert and oxidizing atmospheres at different temperatures are given in Figs. S3 and S4. It can be seen that $C_{12}$ and $C_{13}$ congeners were the dominant carbon homologs for SCCPs compared to raw CP52 (Fig. S1a). The sum fraction of $\mathrm{C}_{10^{-11}}$ congeners under oxidizing condition was higher than that under inert condition. The fraction of $\mathrm{C}_{13}$ congeners slightly decreased, while the sum of $\mathrm{C}_{10-11}$ congeners increased. This indicates that SCCPs underwent carbon chain scission. For MCCPs, $\mathrm{C}_{14}$ was also the predominated congeners, but they showed a noticeable decrease. The decrement of $\mathrm{C}_{14}$ under oxidizing atmosphere was higher than that under inert atmosphere as the temperature 
increased, thus demonstrating the decomposition of MCCPs in the gas phase. With regard to chlorine atom homologs, $\mathrm{Cl}_{7-9}$ congeners were the most abundant congener groups for SCCPs and MCCPs in inert and oxidizing conditions. However, the fraction of $\mathrm{Cl}_{6}$ groups of SCCPs increased at $200{ }^{\circ} \mathrm{C}$, and $\mathrm{Cl}_{5}$ groups were identified as the temperature increased to $300{ }^{\circ} \mathrm{C}$ and above. This indicates that dehydrochlorination in the gas phase enhanced with the increase in temperature. In addition, combustion yields more $\mathrm{Cl}_{5}$ groups than pyrolysis, suggesting that oxygen also facilitated dechlorination. For MCCPs, the fraction of $\mathrm{Cl}_{6-7}$ congeners decreased while higher chlorine congeners $\left(\mathrm{Cl}_{8-10}\right)$ increased with the increase in temperature under oxidizing atmosphere.

For compartment fires or open burning, the temperature field is not uniformly distributed and ranges from hundreds to thousands of degrees centigrade (Stern-Gottfried et al., 2010). The materials that contain CPs were also nonuniformly burned in air or in the absence of oxygen. In this situation, the temperature of these materials can easily reach $200-400^{\circ} \mathrm{C}$, and this process has a high risk of SCCP and MCCP emission into the environment. For example, at low temperatures $\left(\sim 200^{\circ} \mathrm{C}\right)$, inherent SCCPs and MCCPs might be released into water through fire extinguisher actions. At higher temperatures ( $\left.300-400{ }^{\circ} \mathrm{C}\right)$, SCCPs and MCCPs might be released into the atmosphere. Fig. $2 \mathrm{a}-\mathrm{d}$ shows that the total amount of released MCCPs was much higher than that of released SCCPs in both VS and SR. This is consistent with our previous findings that the MCCP concentration was higher than the SCCP concentration in the environment (Zeng et al., 2015).

\subsection{Formation and composition profiles of $C_{9}$ species}

To study the products derived from the decomposition of SCCPs and other CPs, chlorinated hydrocarbons with carbon chain lengths of nine $\left(\mathrm{C}_{9}\right)$ were analyzed qualitatively. The detailed method for qualifying $C_{9}$ congeners can be found in the Supplementary Materials (Part S3, Table S1), and the $\mathrm{C}_{9}$ congeners in VS and SR are shown in Fig. 3. It can be seen that the response of the $C_{9}$ congener in the raw CP52 sample was very low, indicating the low level of $C_{9}$ hydrocarbons. However, high concentrations of $\mathrm{C}_{9}$ hydrocarbons were identified exclusively in the residue at $200^{\circ} \mathrm{C}$, suggesting that the volatilization of $\mathrm{C}_{9}$ was weak. Hence, the weight loss of CP52 was only $\sim 4 \mathrm{wt} \%$ at $200{ }^{\circ} \mathrm{C}$ (Fig. 1a). The peak area of $\mathrm{C}_{9}$ in the residue increased 36 -fold and 140 -fold under inert and oxidative atmospheres, respectively. The results confirmed that CP52 underwent a drastic decomposition at $200{ }^{\circ} \mathrm{C}$, and oxygen facilitated the thermal decomposition of CPs. At $300{ }^{\circ} \mathrm{C}$, the $\mathrm{C}_{9}$ congeners in the gas phase increased continuously, suggesting that the release of
$C_{9}$ into the gas phase was also enhanced with the increase in temperature. In contrast, the $\mathrm{C}_{9}$ congeners in the residue decreased significantly in the presence of oxygen. With a further increase in temperature, the amount of $C_{9}$ congeners decreased in the gas phase and was not detected in the residue.

The $C_{9}$ congener distribution patterns under inert and oxidizing atmospheres are given in Fig. S5. Raw CP52 contains a small amount of $\mathrm{Cl}_{7}$ and $\mathrm{Cl}_{8}$ congeners. However, the peak areas of $\mathrm{Cl}_{7}$ and $\mathrm{Cl}_{8}$ in the residue increased drastically at $200^{\circ} \mathrm{C}$, showing the same trend as for the $\mathrm{C}_{9}$ species. It is noteworthy that pyrolysis led to the formation of more high-chlorinated congeners $\left(\mathrm{Cl}_{7-9}\right)$, whereas combustion generated more low-chlorinated congeners $\left(\mathrm{Cl}_{4-8}\right)$. This demonstrated that oxygen has a stronger effect on the dehydrochlorination of CP52.

Figs. 2 and 3 show that the concentration of SCCPs, MCCPs, and $\mathrm{C}_{9}$ congeners changes coincidentally with an increase in temperature. Although the potential environmental toxicity of $C_{9}$ remains uncertain, it might serve as a precursor of chlorinated compounds during thermal processes. Furthermore, combustion dissociates more chlorine atoms into the gas phase, and the resulting chlorine atoms have the potential to bond with aromatics to unintentionally produce POPs during combustion, such as PCNs, especially under the catalysis of metal (Ryu et al., 2013).

\subsection{Formation and composition profiles of aromatics and chlorinated aromatics}

As a source of carbon, hydrogen, and chlorine, CPs have the potential to generate aromatic and chlorinated aromatic compounds during thermal-related processes. These compounds are generally defined as unintentionally produced POPs (Liu et al., 2016). The raw CP52 was analyzed by GC-QTOF-MS according to the previous procedures. It was found that aromatics and Cl-PAHs were not detected. The aromatics in the VS were mainly divided as follows: substituted benzene (S-Ben), substituted indene (SInde), PAHs, and substituted PAHs (S-PAHs). The substituent groups were mainly aliphatic hydrocarbons (AHCs), such as alkanes and alkenes, with varying carbon chain lengths. Table 1 lists the detectability of SCCPs, $\mathrm{C}_{9}$ species, and aromatic compounds at different temperatures. The detailed composition of PAHs and benzene derivatives is given in Table S2.

The results clearly show the effect of temperature on the release of SCCPs and the formation of aromatic compounds in the gas phase. An increase in the temperature led to the release of SCCPs from the residue into the gas phase. As the temperature exceeded $400{ }^{\circ} \mathrm{C}$, SCCPs and $\mathrm{C}_{9}$ congeners were not detected in either the VS
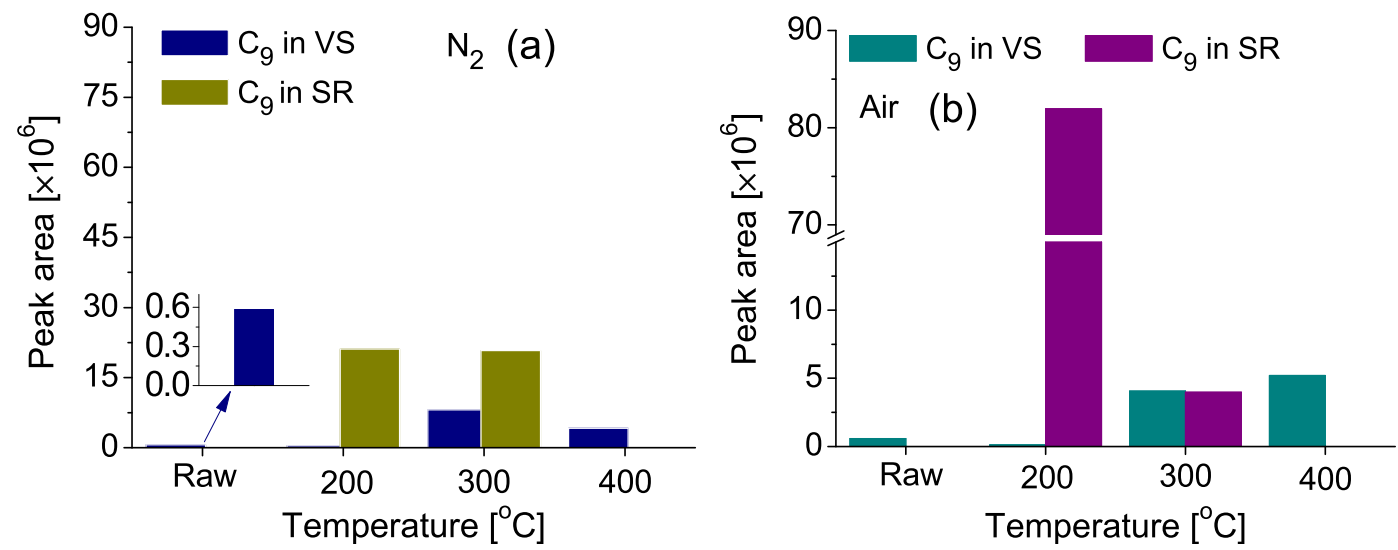

Fig. 3. Total peak areas of C9 hydrocarbons in VS and SR under (a) inert and (b) oxidative atmospheres at different temperatures. 
Table 1

The detectability of SCCPs, MCCPs, $\mathrm{C}_{9}$ congeners, aromatics (PAHs, benzenederivatives) and Cl-PAHs (CBz, PCBs, PCNs).

\begin{tabular}{lllllll}
\hline & \multicolumn{5}{l}{ Temperature $/{ }^{\circ} \mathrm{C}$} \\
\cline { 3 - 7 } & & 200 & 300 & 400 & 600 & 800 \\
\hline SCCPs & Gas phase & + & + & + & nd & nd \\
MCCPs & Residue & + & + & nd & nd & nd \\
C9 species & Gas phase & + & + & + & nd & nd \\
& Residue & + & + & nd & nd & nd \\
PAHs & PAHs & nd & + & + & + & + \\
& S-PAHs & nd & + & + & + & + \\
Benzene derivatives & nd & + & + & + & + \\
CBz & & nd & + & + & + & + \\
PCBs & nd & + & + & + & + \\
PCNs & nd & + & + & + & + \\
\hline
\end{tabular}

“+" compounds detected; "nd" not detected.

or the SR, suggesting that they were transferred from the residue and decomposed in the gas phase. Aromatics and Cl-PAHs were detected at $300{ }^{\circ} \mathrm{C}$ and above, implying that they were formed because of the decrease in SCCP and MCCP concentrations.

\subsubsection{Formation of aromatic hydrocarbons}

The fraction of S-Ben, S-Inde, PAHs and S-PAHs, AHCs in VS is shown in Fig. 4a. Details of the congener distribution patterns of PAHs and S-PAHs as a function of temperature are given in Figs. S6 and S7. The figures show that volatiles were dominated by saturated cycloalkanes and hydrocarbons, and no aromatics were detected at $200{ }^{\circ} \mathrm{C}$, thus demonstrating that CP52 underwent drastic dehydrochlorination and carbon chain cleavage. As the temperature was increased to $300^{\circ} \mathrm{C}$, the amount of AHC decreased considerably. In contrast, a considerable amount of aromatics was quantified, which accounted for approximately $70 \%$ of the total compounds detected. This indicated that aromatization occurred among the unsaturated structures in the gas phase. These aromatics were mostly substituted congeners such as S-Ben, S-Inde, and S-PAHs, and the amount of these aromatics was much higher than that of PAHs.

According to the temperature-dependent ring distribution of aromatic hydrocarbons (Fig. 4b), 1- to 2-ring aromatics occupied the largest proportions from inert and oxidizing decomposition of $\mathrm{CP} 52$ at $300^{\circ} \mathrm{C}$. The decomposition of CP52 under inert atmosphere generated more S-Ben but less S-PAHs and a negligible amount of PAHs compared with that under oxidative atmosphere at $300{ }^{\circ} \mathrm{C}$ (Fig. 4a). This implies that oxygen can also promote the ring growth reaction to generate 2-ring PAHs, e.g., from benzene and indene to naphthalene and acenaphthene. An increase in the temperature from $300{ }^{\circ} \mathrm{C}$ to $600{ }^{\circ} \mathrm{C}$ produced a continuous decrease in the amount of S-Ben but a substantial increase in the amount of PAHs and S-PAHs. It seems that S-Ben can be regarded as the intermediate or precursor of PAHs and S-PAHs (Froese and Hutzinger, 1997). The increase in the amount of PAHs was mainly attributed to the dramatic increase in 3-ring aromatics. As the temperature exceeded $600{ }^{\circ} \mathrm{C}$, the amount of PAH substantially increased but that of SPAHs remarkably decreased. It should be noted that the increase in 3- to 5-ring PAHs was due to the decrease in the amount of 2-ring PAHs. The above results indicate that the thermal decomposition of CP52 can be the source of aromatics in environment matrices.

\subsubsection{Formation of $\mathrm{Cl}-\mathrm{PAHS}$}

Typical Cl-PAHs in the gas phase, such as CBz, PCBs, and PCNs, were analyzed quantitatively. The total mass concentration and homologue compositions of $\mathrm{CBz}, \mathrm{PCBs}$, and $\mathrm{PCNs}$ are shown in Fig. 5. It should be noted that PCDD/Fs were not detected in any experiment in this study. Although $\mathrm{CBz}$ is generally regarded as the chlorinated precursor of PCDD/Fs through heterogeneous catalyzed reactions (Stanmore, 2004), it is unlikely to occur because CP52 is ash free. Nevertheless, industrial products or mixtures of solid waste will certainly contain ash or catalytic metal particle, which implies that CP52 has a high potential to produce PCDD/Fs in the industrial process. Therefore, firing, open burning, or incineration of materials laden with CPs might contribute to the emission of $\mathrm{PCDD} / \mathrm{Fs}$ in the environment.

Fig. 5 clearly shows that the dominant congeners of $\mathrm{CBz}$ were low chlorinated benzenes, such as mono-chlorobenzene (MCBz) and dichlorobenzene (DiCBz). The total concentration of $\mathrm{CBz}$, as well as that of $\mathrm{MCBz}$ and $\mathrm{DiCBz}$, slightly increased at $200-400{ }^{\circ} \mathrm{C}$ and increased dramatically at $600{ }^{\circ} \mathrm{C}$. Surprisingly, the change in $\mathrm{CBz}$ concentration with temperature was opposite to that in SCCP concentration (Fig. 2a-b), thus demonstrating a close relationship between the decomposition of CPs and the formation of $\mathrm{CBz}$. The concentration of $\mathrm{MCBz}$ and $\mathrm{DiCBz}$ reached a maximum value at $600{ }^{\circ} \mathrm{C}$, and it decreased remarkably as the temperature was increased further. The thermal decomposition of 1,3-dichloropropene also obtained the highest yield of mono- and di-CBz at $527-677^{\circ} \mathrm{C}$, which was in accordance with that observed by Ahubelem et al. (2015). These results suggest that $\mathrm{CBz}$ can be formed from the small molecules derived from CP52 decomposition. The above results indicate that high temperatures can minimize the formation and release of $\mathrm{CBz}$ without ash. The concentration of $\mathrm{CBz}$ under inert and oxidizing atmosphere was very similar at $200-400{ }^{\circ} \mathrm{C}$, implying that oxygen has little effect on the formation of $\mathrm{CBz}$ at low temperature. However, the
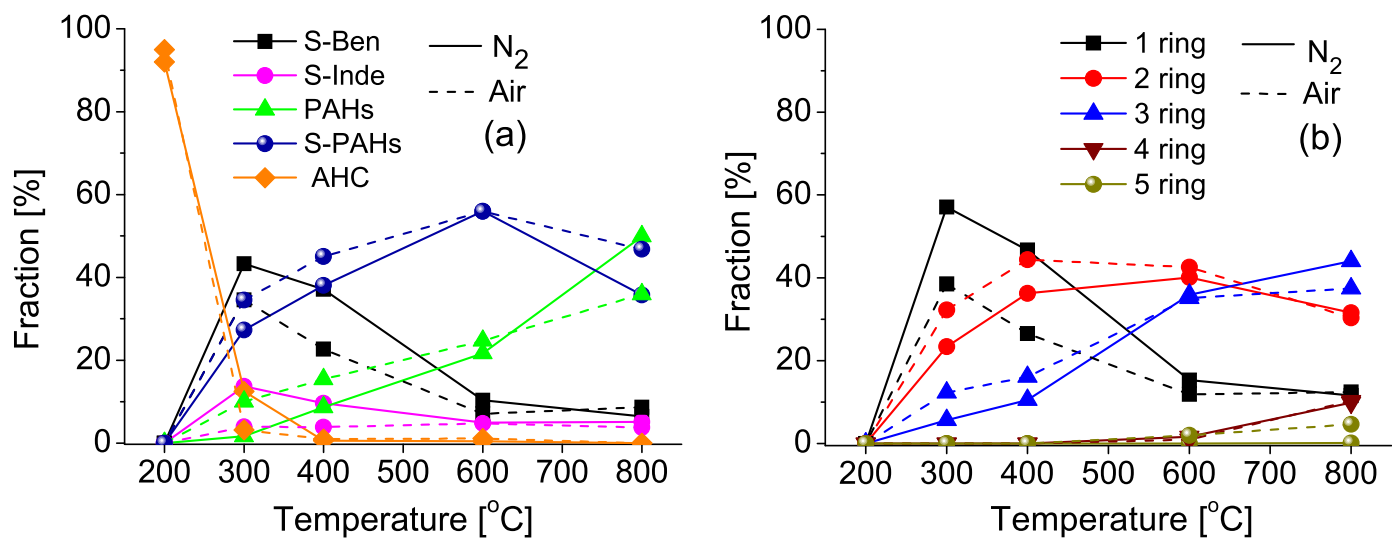

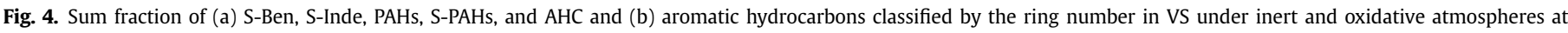
different temperatures. Solid line denotes inert atmosphere, and dashed line represents oxidizing atmosphere. 

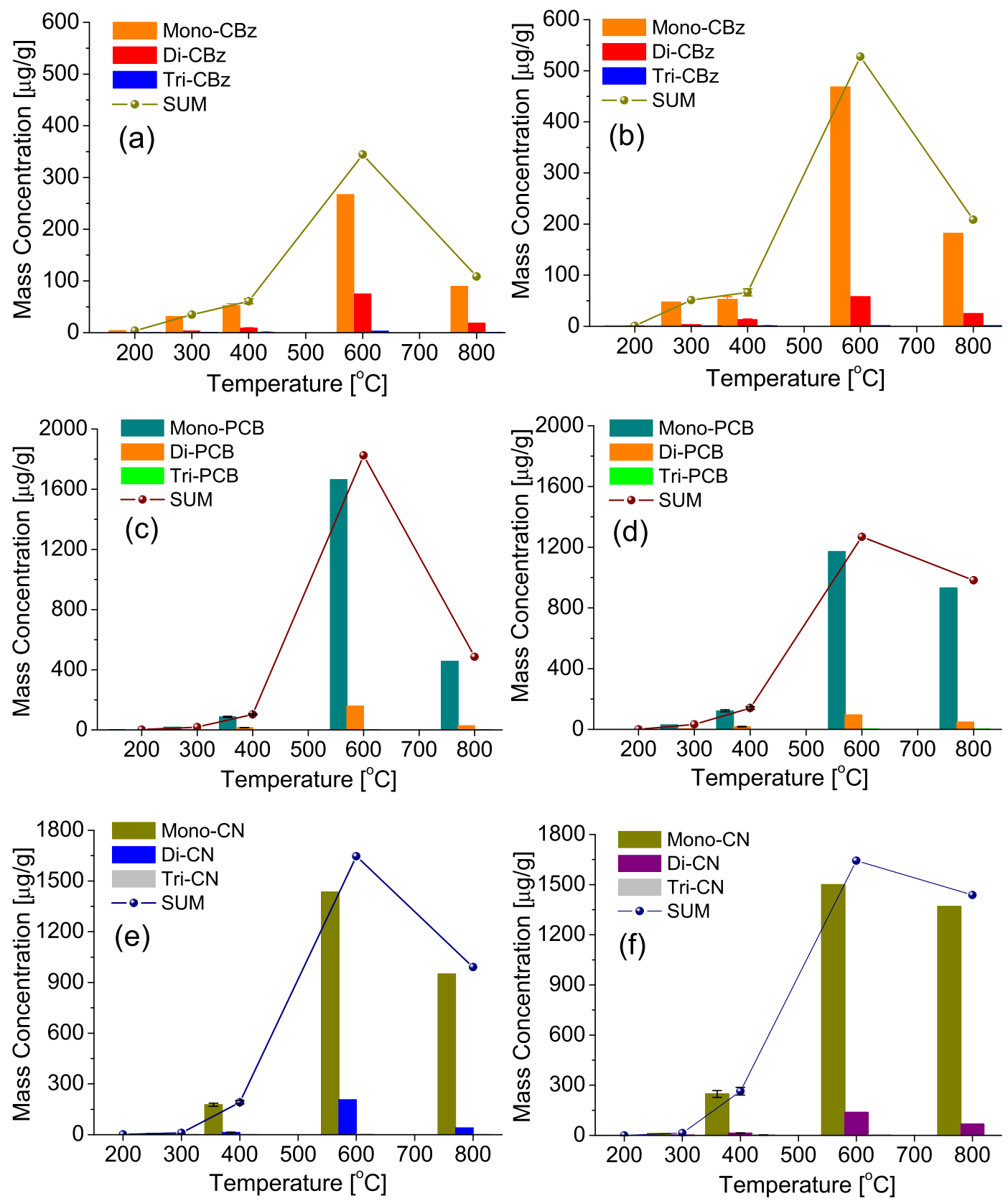

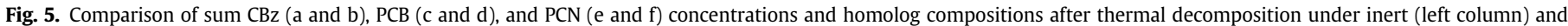
oxidative atmospheres (right column) at different temperatures.

concentration of $\mathrm{CBz}$ from combustion, especially that of $\mathrm{MCBz}$, was much higher than that from pyrolysis at higher temperatures $\left(>400{ }^{\circ} \mathrm{C}\right)$.

PCB and PCN concentrations in the gas phase showed a similar trend to that of CBz. PCBs and PCNs were also dominated by monoand di-congeners, which suggests that PCBs and PCNs might be formed through similar chemical routes and are related to the formation of CBz (Oh et al., 2007; Jiang et al., 2015). Accordingly, at 200-400 ${ }^{\circ} \mathrm{C}, \mathrm{PCB}$ and $\mathrm{PCN}$ concentrations did not result in a substantial difference in $\mathrm{CBz}$ concentration in the gas phase. However, an increase in temperature led to a considerable increase in PCB and $\mathrm{PCN}$ concentrations, which was over two times higher than $\mathrm{CBz}$ concentration. At $800{ }^{\circ} \mathrm{C}$, the oxidative destruction or dechlorination of PCBs and PCNs may result in a decrease in their concentration. As a result, the amount of PAHs in the gas phase greatly increased.

\section{Conclusions}

The thermal decomposition characteristics of CP52 under inert and oxidative atmospheres were investigated by TGA, and the resulting products were analyzed by GC-QTOF-HRMS. It was found that the thermal decomposition of CPs would release SCCPs and MCCPs into the environment. Moreover, aromatics and $\mathrm{Cl}-\mathrm{PAHs}$ could also be produced unintentionally during this process. In general, SCCPs were gradually released into the gas phase at temperatures lower than $400^{\circ} \mathrm{C}$, and considerable amounts of aromatic and $\mathrm{Cl}-\mathrm{PAHs}$ were formed at higher temperatures. The present study demonstrated the synergistic emission of SCCPs and Cl-PAHs during the thermal processes of CPs such as incineration, open 
burning, or accidental fire in a department. Higher temperature can minimize the formation of SCCPs, but it will generate Cl-PAHs. Although the results cannot be applied directly to the thermal behaviors of materials laden with CPs, they provide a brief understanding about the emission and transformation of SCCPs and their synergistic formation with Cl-PAHs during thermal processes.

\section{Conflict of interest}

The authors declare that there are no conflicts of interest.

\section{Acknowledgments}

We thank the National Natural Science Foundation of China (21625702, 21337002, and 21321004), the National Basic Research Program of China (2015CB453102), and the Strategic Priority Research Program of the Chinese Academy of Science (XDB14010400) for financial support.

\section{Appendix A. Supplementary data}

Supplementary data related to this article can be found at http:// dx.doi.org/10.1016/j.chemosphere.2017.07.019.

\section{References}

Ahubelem, N., Shah, K., Moghtaderi, B., Altarawneh, M., Dlugogorski, B.Z., Page, A.J., 2015. Formation of chlorobenzenes by oxidative thermal decomposition of $1,3-$ dichloropropene. Combust. Flame 162, 2414-2421.

Bayen, S., Obbard, J.P., Thomas, G.O., 2006. Chlorinated paraffins: a review of analysis and environmental occurrence. Environ. Int. 32, 915-929.

Bergman, A., Hagman, A., Jacobsson, S., Jansson, B., Ahlman, M., 1984. Thermal degradation of polychlorinated alkanes. Chemosphere 13, 237-250.

Camino, G., Costa, L., 1980. Thermal degradation of a highly chlorinated paraffin used as a fire retardant additive for polymers. Polym. Degrad. Stab. 2, 23-33.

Camino, G., Costa, L., 1981. Thermal degradation of polymer-fire retardant mixtures-Part I: polypropylene-chlorinated paraffin. Evidence of interactions. Polym. Degrad. Stab. 3, 423-430.

Cao, Y., Harada, K.H., Hitomi, T., Niisoe, T., Wang, P., Shi, Y., Yang, H.-R., Takasuga, T., Koizumi, A., 2017. Lactational exposure to short-chain chlorinated paraffins in China, Korea, and Japan. Chemosphere 173, 43-48.

Castro, A., Soares, D., Vilarinho, C., Castro, F., 2012. Kinetics of thermal dechlorination of PVC under pyrolytic conditions. Waste Manag. 32, 847-851.

Diaz Silvarrey, L.S., Phan, A.N., 2016. Kinetic study of municipal plastic waste. Int. J. Hydrogen Energy 41, 16352-16364.

Diefenbacher, P.S., Bogdal, C., Gerecke, A.C., Glüge, J., Schmid, P., Scheringer, M., Hungerbühler, K., 2015. Short-chain chlorinated paraffins in Zurich, Switzerland-atmospheric concentrations and emissions. Environ. Sci. Technol. 49, 9778-9786.

Feo, M.L., Eljarrat, E., Barceló, D., 2009. Occurrence, fate and analysis of polychlorinated n-alkanes in the environment. TrAC Trends Anal. Chem. 28, $778-791$.

Froese, K.L., Hutzinger, O., 1997. Mechanisms of the formation of polychlorinated benzenes and phenols by heterogeneous reactions of C2 aliphatics. Environ. Sci. Technol. 31, 542-547.

Gao, W., Wu, J., Wang, Y., Jiang, G., 2016a. Distribution and congener profiles of short-chain chlorinated paraffins in indoor/outdoor glass window surface films and their film-air partitioning in Beijing, China. Chemosphere 144, 1327-1333.

Gao, W., Wu, J., Wang, Y.W., Jiang, G.B., 2016b. Quantification of short- and mediumchain chlorinated paraffins in environmental samples by gas chromatography quadrupole time-of-flight mass spectrometry. J. Chromatogr. A 1452, 98-106.

Hilger, B., Fromme, H., Volkel, W., Coelhan, M., 2013. Occurrence of chlorinated paraffins in house dust samples from Bavaria, Germany. Environ. Pollut. 175, $16-21$.

Houde, M., Muir, D.C.G., Tomy, G.T., Whittle, D.M., Teixeira, C., Moore, S., 2008 Bioaccumulation and trophic magnification of short- and medium-chain chlorinated paraffins in food webs from lake Ontario and lake Michigan. Environ. Sci. Technol. 42, 3893-3899.

Iino, F., Takasuga, T., Senthilkumar, K., Nakamura, N., Nakanishi, J., 2005. Risk assessment of short-chain chlorinated paraffins in Japan based on the first market basket study and species sensitivity distributions. Environ. Sci. Technol. 39, 859-866.

Jiang, W., Huang, T., Mao, X., Wang, L., Zhao, Y., Jia, C., Wang, Y., Gao, H., Ma, J., 2017. Gridded emission inventory of short-chain chlorinated paraffins and its validation in China. Environ. Pollut. 220 (Part A), 132-141.

Jiang, X., Liu, G., Wang, M., Zheng, M., 2015. Fly ash-mediated formation of polychlorinated naphthalenes during secondary copper smelting and mechanistic aspects. Chemosphere 119, 1091-1098.

Jordan, K.J., Suib, S.L., Koberstein, J.T., 2001. Determination of the degradation mechanism from the kinetic parameters of dehydrochlorinated poly(vinyl chloride) decomposition. J. Phys. Chem. B 105, 3174-3181.

Kaimal, V.K., Vijayabalan, P., 2016. A study on synthesis of energy fuel from waste plastic and assessment of its potential as an alternative fuel for diesel engines. Waste Manag. 51, 91-96.

Liu, G., Zheng, M., Jiang, X., Jin, R., Zhao, Y., Zhan, J., 2016. Insights into the emission reductions of multiple unintentional persistent organic pollutants from industrial activities. Chemosphere 144, 420-424.

Ma, X., Zhang, H., Zhou, H., Na, G., Wang, Z., Chen, C., Chen, J., Chen, J., 2014a. Occurrence and gas/particle partitioning of short- and medium-chain chlorinated paraffins in the atmosphere of Fildes Peninsula of Antarctica. Atmos. Environ. 90, 10-15.

Ma, X.D., Zhang, H.J., Wang, Z., Yao, Z.W., Chen, J.W., Chen, J.P., 2014b. Bioaccumulation and trophic transfer of short chain chlorinated paraffins in a marine food web from Liaodong Bay, North China. Environ. Sci. Technol. 48 5964-5971.

Oh, J.-E., Gullett, B., Ryan, S., Touati, A., 2007. Mechanistic relationships among PCDDs/fs, PCNs, PAHs, ClPhs, and ClBzs in municipal waste incineration. Environ. Sci. Technol. 41, 4705-4710.

Poddar, N.B., Thomas, S., Wornat, M.J., 2013. Polycyclic aromatic hydrocarbons from the co-pyrolysis of 1,3-butadiene and propyne. Proc. Combust. Inst. 34, 1775-1783.

POPRC (Persistent Organic Pollutants Review Committee), 2017. Recommendation by the Persistent Organic Pollutants Review Committee to List Short-Chain Chlorinated Paraffins in Annex A to the Convention (UNEP/POPS/COP.8/14).

Ryu, J.-Y., Kim, D.-H., Jang, S.-H., 2013. Is chlorination one of the major pathways in the formation of polychlorinated naphthalenes (PCNs) in municipal solid waste combustion? Environ. Sci. Technol. 47, 2394-2400.

Sosa, J.M., 1975. Thermal stability of chlorinated paraffins. J. Polym. Sci. Polym. Chem. Ed. 13, 2397-2405.

Stanmore, B.R., 2004. The formation of dioxins in combustion systems. Combust Flame 136, 398-427.

Stern-Gottfried, J., Rein, G., Bisby, L.A., Torero, J.L., 2010. Experimental review of the homogeneous temperature assumption in post-flashover compartment fires. Fire Saf. J. 45, 249-261.

Tang, Y., Ma, X., Lai, Z., Fan, Y., 2015. Thermogravimetric analyses of co-combustion of plastic, rubber, leather in N2/O2 and CO2/O2 atmospheres. Energy 90 (Part 1), 1066-1074.

Thompson, R., Vaughan, M., 2014. Medium-chain chlorinated paraffins (MCCPs): a review of bioaccumulation potential in the aquatic environment. Integr. Environ. Assess. Manag. 10, 78-86.

van der Gon, H.D., van het Bolscher, M., Visschedijk, A., Zandveld, P., 2007. Emissions of persistent organic pollutants and eight candidate POPs from UNECE-Europe in 2000, 2010 and 2020 and the emission reduction resulting from the implementation of the UNECE POP protocol. Atmos. Environ, 41, 9245-9261.

van Mourik, L.M., Gaus, C., Leonards, P.E.G., de Boer, J., 2016. Chlorinated paraffins in the environment: a review on their production, fate, levels and trends between 2010 and 2015. Chemosphere 155, 415-428.

Wang, X.-T., Xu, S.-Y., Wang, X.-K., Hu, B.-P., Jia, H.-H., 2017. Occurrence, homologue patterns and source apportionment of short- and medium-chain chlorinated paraffins in suburban soils of Shanghai, China. Chemosphere 180, 302-311.

Xia, D., Gao, L., Zheng, M., Li, J., Zhang, L., Wu, Y., Tian, Q., Huang, H., Qiao, L., 2016. Human exposure to short- and medium-chain chlorinated paraffins via mothers' milk in Chinese Urban population. Environ. Sci. Technol. 51, 608-615.

Xu, C., Xu, J., Zhang, J., 2014. Emission inventory prediction of short chain chlorinated paraffins (SCCPs) in China. Acta Sci. Nat. Univ. Pekin. 50, 369-378.

Yu, J., Sun, L., Ma, C., Qiao, Y., Yao, H., 2016. Thermal degradation of PVC: a review. Waste Manag. 48, 300-314.

Zeng, L., Lam, J.C.W., Wang, Y., Jiang, G., Lam, P.K.S., 2015. Temporal trends and pattern changes of short- and medium-chain chlorinated paraffins in marine mammals from the South China Sea over the past decade. Environ. Sci. Technol. 49, 11348-11355.

Zeng, L., Wang, T., Wang, P., Liu, Q., Han, S., Yuan, B., Zhu, N., Wang, Y., Jiang, G., 2011 Distribution and trophic transfer of short-chain chlorinated paraffins in an aquatic ecosystem receiving effluents from a sewage treatment plant. Environ. Sci. Technol. 45, 5529-5535.

Zeng, L.X., Wang, T., Ruan, T., Liu, Q., Wang, Y.W., Jiang, G.B., 2012. Levels and distribution patterns of short chain chlorinated paraffins in sewage sludge of wastewater treatment plants in China. Environ. Pollut. 160, 88-94.

Zhang, F., Wang, H., Zhang, L., Zhang, J., Fan, R., Yu, C., Wang, W., Guo, Y., 2014a. Suspected-target pesticide screening using gas chromatography-quadrupole time-of-flight mass spectrometry with high resolution deconvolution and retention index/mass spectrum library. Talanta 128, 156-163.

Zhang, H., Gao, Y., Ma, X., Geng, N., Zhang, Y., Chen, J., 2013. Reach advances in analytical methods, environmental behaviors and toxic effects of short chain chlorinated paraffins (SCCPs). Sci. Sin. Chim. 43, 255-264.

Zhang, H., Wang, Y., Sun, C., Yu, M., Gao, Y., Wang, T., Liu, J., Jiang, G., 2014b. Levels and distributions of hexachlorobutadiene and three chlorobenzenes in biosolids from wastewater treatment plants and in soils within and surrounding a chemical plant in China. Environ. Sci. Technol. 48, 1525-1531. 